\title{
Electron spin relaxation due to reorientation of a permanent zero field splitting tensor
}

\author{
Nathaniel Schaefle and Robert Sharp \\ Department of Chemistry, University of Michigan, Ann Arbor, Michigan 48109
}

(Received 1 June 2004; accepted 2 July 2004)

\begin{abstract}
Electron spin relaxation of transition metal ions with spin $S \geqslant 1$ results primarily from thermal modulation of the zero field splitting (zfs) tensor. This occurs both by distortion of the zfs tensor due to intermolecular collisions and, for complexes with less than cubic symmetry, by reorientational modulation of the permanent zfs tensor. The reorientational mechanism is much less well characterized in previous work than the distortional mechanism although it is an important determinant of nuclear magnetic resonance (NMR) paramagnetic relaxation enhancement phenomena (i.e., the enhancement of NMR relaxation rates produced by paramagnetic ions in solution or NMR-PRE). The classical density matrix theory of spin relaxation does not provide an appropriate description of the reorientational mechanism at low Zeeman field strengths because the zero-order spin wave functions are stochastic functions of time. Using spin dynamics simulation techniques, the time correlation functions of the spin operators have been computed and used to determine decay times for the reorientational relaxation mechanism for $S=1$. In the zfs limit of laboratory field strengths $\left(H_{\text {Zeem }} \ll H_{\mathrm{zfs}}^{\circ}\right)$, when the zfs tensor is cylindrical, the spin decay is exponential, the spin relaxation time, $\tau_{S}^{\circ} \approx 0.53 \tau_{R}^{(1)}$, where $\tau_{R}^{(1)}$ is the reorientational correlation time of a molecule-fixed vector. The value of $\tau_{S}^{\circ}$ is independent of the magnitude of the cylindrical zfs parameter $(D)$, but it depends strongly on low symmetry zfs terms (the $E / D$ ratio). Other spin dynamics (SD) simulations examined spin decay in the intermediate regime of field strengths where $H_{\text {Zeem }} \approx H_{\text {zfs }}^{\circ}$, and in the vicinity of the Zeeman limit. The results demonstrate that the reorientational electron spin relaxation mechanism is often significant when $H_{\mathrm{zfs}}^{\circ} \geqslant H_{\mathrm{Zeem}}$, and that its neglect can lead to serious errors in the interpretation of NMR-PRE data. (C) 2004 American Institute of Physics. [DOI: 10.1063/1.1786577]
\end{abstract}

\section{INTRODUCTION}

Electron spin relaxation of transition metal ions with spin $S \geqslant 1$ results primarily from thermal modulation of the zero field splitting (zfs) tensor. In a seminal study, Van Vleck $^{1}$ described the relaxation mechanism in solids as resulting from phonon modulation of the zfs tensor. Bloembergen and Morgan ${ }^{2}$ subsequently extended this concept to spin relaxation of hexaquated metal cations in aqueous solution, proposing a mechanism based on collisional distortion of the metal zfs tensor that results from Brownian motion in the solution phase. The systems they considered $\left[\mathrm{Me}\left(\mathrm{H}_{2} \mathrm{O}\right)_{6}^{2+}\right.$ cations with $S \geqslant 1$ ] lack a permanent zfs tensor because of the octahedral site symmetry of the metal ion. In the much more common case where the metal ion has noncubic site symmetry, a permanent zfs interaction, described by a Hamiltonian $H_{\mathrm{zfs}}^{\circ}(t)$ is present. The zfs tensor may be modulated both by collisional distortion and by Brownian reorientation. ${ }^{3}$ In the common situation where reorientation proceeds by a sequence of small steps, these processes are uncorrelated and additive. The present study is an analysis of electron spin relaxation due to reorientation of the permanent zfs tensor. The mechanism associated with collisional distortion of a zfs tensor has been described in previous work. ${ }^{4-13}$

The electron spin motion is assumed to be driven by a Hamiltonian which is a sum of Zeeman and permanent zfs terms,

$$
H_{S}^{\circ}=H_{Z}+H_{\mathrm{zfs}}^{\circ}(t),
$$

the latter time dependent due to Brownian reorientation. Electron spin relaxation is defined as the process of thermal decay of time correlation functions (TCFs), of the spin operators, for example,

$$
G_{Z}(t) \equiv \overline{\left\langle S_{Z}(0) S_{Z}(t)\right\rangle} .
$$

The angular brackets represent an expectation value over the spin degrees of freedom, and the superscripting line denotes a thermal average over molecular degrees of freedom. In some physical situations (but by no means all), relaxation is exponential in time, and it is then appropriate to define spin relaxation times $\tau_{S, r}$, where $r$ labels the spatial polarization. When spin relaxation is nonexponential, the decay envelope can be described less precisely in terms of a (1/e) decay time $\tau_{S, r}^{(e)}$.

According to Eq. (1), the electron spin motion can be described with respect to two limiting situations which occur when one of the terms on the right-hand side (rhs.) of Eq. (1) is much larger than the other. The limit of large Zeeman field strength $H_{Z} \gg H_{\mathrm{zfs}}^{\circ}$ is usually termed the Zeeman limit, and the opposite situation $H_{Z} \ll H_{\mathrm{zfs}}^{\circ}$, the zfs limit. The zfs limit requires, additionally, that reorientation not be so fast that the zfs level structure is collapsed. An analysis of the spin motion in the vicinity of the Zeeman limit is relatively straight- 
forward, since in that case, the zero-order spin wave functions are eigenfunctions of $H_{Z}$ and are not explicitly time dependent. Electron spin relaxation can then be formulated in the Zeeman basis using Redfield Theory, with $H_{\mathrm{zfs}}^{\circ}(t)$ as a perturbation. This approach has been used ${ }^{14-19}$ to calculate the reorientational contribution to (esr) linewidths for the half-integer spin ions, $\mathrm{Cr}^{3+}(S=3 / 2), \mathrm{Mn}^{2+}(S=5 / 2)$, and $\mathrm{Gd}^{3+}(S=7 / 2)$, for which the permanent zfs is relatively small.

Relaxation in the vicinity of the zfs limit is more difficult to treat because the zero-order Hamiltonian $H_{\mathrm{zfs}}^{\circ}(t)$ is explicitly time dependent, and it is the consequence of this time dependence that we wish to describe. In the absence of reorientation, the zfs-limit wave functions are the eigenfunctions of $H_{\mathrm{zfs}}^{\circ}(t)$, which are spatially quantized (or polarized) along the principal axes of the molecule-fixed zfs tensor, rather than along the laboratory field. The physical situation becomes more complex still when $H_{\mathrm{zfs}}^{\circ}(t)$ fluctuates due to Brownian reorientation as well as when $H_{Z}=H_{\mathrm{zfs}}^{\circ}$ (the intermediate regime). In these cases, the spin wave functions lack well-defined spatial polarization and are stochastic functions of time.

The objective of this work is to develop techniques suitable for calculating the TCFs of Eq. (2) at all laboratory field strengths in the situation where $H_{\mathrm{zfs}}^{\circ}(t)$ is modulated by Brownian reorientation. In particular, we wish to determine the situations in which spin decay is exponential, and whether, in those cases, a relaxation time can be estimated from the static zfs parameters $(D, E$, etc. $)$ and the reorientational correlation times. To simulate the electron spin motions, SD techniques, which treat the electron spin motion quantum mechanically and molecular reorientation classically, were used.

The results of the study extend our understanding of NMR paramagnetic relaxation enhancement (NMR-PRE) phenomena, (i.e., the enhancements of $R_{1}$ and $R_{2}$ NMR relaxation rates that are produced by dissolved paramagnetic ions in solution). This field is currently very active, ${ }^{20}$ particularly in regard to the development of new MRI contrast agents and to the optimization of relaxivity. ${ }^{21}$ Experimentally, NMR-PRE data are collected across a wide range of field strengths, typically, 0.001-2 T. For many complexes, the low field region corresponds to the zfs limit, and an understanding of electron spin relaxation in this situation is needed. The collisional zfs distortion mechanism has been analyzed previously, ${ }^{4-13}$ both in the low field and intermediate regimes, but the zfs reorientational mechanism is less well understood. The objective of this study is to further characterize the reorientational mechanism for $S=1$.

The description of NMR-PRE phenomena in physical situations where zfs interactions are large and molecular reorientation is rapid requires rather complex calculations of the Laplace-Fourier transform of the TCF of the nuclearelectron dipolar interaction. Pioneering work in this area is due to Benetis, Kowalewski, Westlund and their co-workers, ${ }^{22-26}$ who, in the 1980 s, developed the stochastic Liouville formalism for calculating NMR-PRE. Our approach to the same problem uses SD simulation methods, the current implementation of which is described below. In the present study, SD simulation is used to describe the timedomain motions of two TCFs, namely: (1) the spin TCF of Eq. (2), the decay of which (even when nonexponential) constitutes electron spin relaxation; and (2) the TCF of the electron-nuclear dipolar interaction, the low frequency part of which is proportional to the NMR-PRE.

\section{THEORY}

The Hamiltonians on the rhs. of Eq. (1) are in general noncommuting and can be written, for $S=1$, in the form,

$$
\begin{aligned}
& H_{Z}=g_{e} \beta_{e} B_{0} S_{Z}, \\
& H_{\mathrm{zfs}}^{\circ}=h c D\left[\hat{S}_{z}^{2}-S(S+1) / 3\right]+h c E\left(\hat{S}_{x}^{2}-\hat{S}_{y}^{2}\right) .
\end{aligned}
$$

Equations (3) and (4) are expressed in different coordinate frames. $H_{Z}$ is written in the laboratory frame with $Z$ parallel to the Zeeman field $B_{0} ; g_{e}, \beta_{e} h, c$ are the electron $g$-value, the Bohr magneton, Planck's constant, and the speed of light. $H_{\mathrm{zfs}}^{\circ}$ is written in the molecule-fixed zfs principal axis system, indicated by a superscripting karat on the spin variables. Although the zfs parameters $D$ and $E$ are time independent, $H_{\mathrm{zfs}}^{\circ}(t)$ is time dependent due to Brownian reorientation of the zfs tensor. For spin $S \geqslant 2$, Eq. (4) contains additional higher order terms in the spin operators, but for $S \leqslant 3 / 2$, these vanish by the dimensionality of the spin system.

\section{A. The spin TCFs}

The TCFs of Eq. (2) can be evaluated as the trace across any interval $t_{0}-t^{\prime}$, where $H_{S}$ is time independent,

$$
G_{Z}\left(t^{\prime}-t_{0}\right)=\operatorname{Tr}\left\{\rho_{S}^{\circ} S_{Z} U^{\dagger}\left(t^{\prime}, t_{0}\right) S_{Z} U\left(t^{\prime}, t_{0}\right)\right\},
$$

where $U\left(t^{\prime}, t_{0}\right)$ is the spin propagator,

$$
U\left(t^{\prime}, t_{0}\right)=\exp \left[-i H_{S}\left(t_{0}\right)\left(t^{\prime}-t_{0}\right) / \hbar\right],
$$

and $\rho_{S}^{\circ}$ is the density operator of the electron spin system. In NMR experiments, the electron spin system is at thermal equilibrium, i.e.,

$$
\begin{aligned}
\rho_{S} & =\rho_{S}^{\circ} \\
& =\exp \left(-\overline{H_{S}} / k T\right) .
\end{aligned}
$$

The Hamiltonian of Eq. (1) $H_{S}$, depends on both spin and molecular degrees of freedom. The superscripting line of Eq. (7b) indicates an ensemble average over the molecular coordinates. When the spin system is in the high temperature limit, Eq. (7b) reduces to

$$
\rho_{S}^{\circ}=(2 S+1)^{-1} \underline{\underline{1}} \text {. }
$$

Equation (2) can be evaluated by simulating molecular reorientation as a random walk process in the space of the Euler angles which describe molecular orientation. Our implementation of this model (a FORTRAN program called PARELAX $2^{27}$ ) follows the work of Ivanov ${ }^{28}$ and is based on the following assumptions: (1) molecular reorientation results from a sequence of rotational jumps which occur at randomly spaced intervals; (2) individual jumps are rapid compared to the inverse transition frequencies of the spin system; (3) the rotation axes of individual jumps are oriented randomly in space; (4) the magnitude of the jump angle is 
distributed as a Gaussian deviate of width $\sigma_{\phi}$ and zero mean. Thus the molecular motion is described as a thermal ensemble of trajectories, each consisting of a sequence of intervals of random duration, $\left(t_{1}, t_{2}, t_{3}, \ldots\right)$ connected by sudden rotational jumps. The description of molecular motions is much less detailed than that provided by full molecular dynamics (MD) simulation, ${ }^{29}$ but the model appears well suited to computation of spin TCFs. The reorientational correlation time $\tau_{R}^{(1)}$ of a molecule-fixed vector is determined by two parameters $\sigma_{\phi}$ and $n_{J}$, where $n_{J}$ is the average number of jumps per $\tau_{R}^{(1)}$ interval (the quantities $\tau_{R}^{(1)}$ and $n_{J}$ are treated as input parameters of the calculation). In trial simulations, we have confirmed the approximate relation, ${ }^{30}$

$$
\sigma_{\phi}=\pi \sqrt{0.101 / n_{J}}
$$

from which $\sigma_{\phi}$ is calculated.

The Hamiltonian in Eq. (6) is time independent in the intervals between jumps and changes suddenly during jumps. The propagator can be decomposed as follows:

$$
\begin{aligned}
U\left(t, t_{0}\right)= & U^{(0)}\left(t_{1}, t_{0}\right) U^{\prime}\left(t_{1}\right) U^{(1)}\left(t_{2}, t_{1}\right) \\
& \times U^{\prime}\left(t_{2}\right) \ldots U^{(n)}\left(t, t_{n}\right) .
\end{aligned}
$$

$U^{(n)}\left(t, t_{n}\right)$ is the propagator in the interval $t_{n}-t_{n+1}$, and $U^{\prime}\left(t_{n}\right)$ is the propagator for the jump connecting the intervals, $n$ and $n+1$. If jumps are rapid, (i.e., the time scale of the jump is rapid compared to the inverse transition frequencies of $H_{S}$ ), the state vector is unaffected (this is the "Sudden Approximation,"31,32):

$$
U^{\prime}\left(t_{n}\right)=\underline{\underline{1}} .
$$

Spin dynamics simulations evaluate $G_{r}(t)$ as an ensemble average of random walk trajectories constructed in this way. At the beginning of the $n$th interval, the spin Hamiltonian is computed and the propagator $U^{(n)}\left(t, t_{n}\right)$ evaluated from the series definition. Then the spin TCF is evaluated at a sequence of time steps within the interval until the next jump occurs, when new values of $H_{S}$ and $U^{(n+1)}\left(t, t_{n+1}\right)$ are evaluated. This calculation requires that the new spin Hamiltonian, which is expressed in the MF (molecule-fixed coordinate frame) in Eq. (5), be reexpressed in the LF (laboratory frame). For this purpose, the spin operators are transformed in the spherical basis using Wigner rotation matrix elements, $D_{q, q^{\prime}}^{\ell}(\alpha, \beta, \gamma)$,

$$
\hat{S}_{q}^{(2)}=\sum_{q^{\prime}=-2}^{+2} S_{q^{\prime}}^{(2)} D_{q, q^{\prime}}^{(2)}(\alpha, \beta, \gamma),
$$

where $(\alpha, \beta, \gamma)$ are the Euler angles of the transformation.

It is important in these calculations that the propagator be evaluated as accurately as possible in order that the spin operators retain their norm after many propagation steps. This can be done satisfactorily using double precision variables as long as the time step used in Eq. (6) is not too long. In practice, we set the fundamental time step of the trajectory equal to $\left(7^{-1} h /\left\langle H_{S}\right\rangle_{m}\right)$, where $\left\langle H_{S}\right\rangle_{m}$ is the largest matrix element of $\left\langle H_{S}\right\rangle$. In this way, seven time steps describe one period of the fastest oscillation of the coherent spin motion. (Note that an interval between rotational jumps usually con- tains several time steps of the trajectory.) This algorithm is stable and accurate across trajectories consisting of $>10^{4}$ time steps.

\section{B. Dipolar TCFs}

In addition to the spin TCFs, we are also interested in the dipolar TCFs, $G_{\text {dip }}(t)$, for the electron-nuclear $(S-I)$ dipolar interaction that is responsible for NMR-PRE. These quantities are defined as follows: ${ }^{33}$

The $S$ - $I$ dipolar Hamiltonian can be written in spherical tensor form as

$$
\begin{aligned}
& H_{\mathrm{dip}}=3^{-1 / 2} \hbar \kappa r_{I S}^{-3} \sum_{q=-1}^{+1}(-1)^{1-q} I_{q}^{(1)} F_{-q}^{(1)}(\theta, \varphi), \\
& \kappa=-30^{1 / 2} \gamma_{I} g_{S} \beta_{e}\left(\mu_{0} / 4 \pi\right) .
\end{aligned}
$$

In Eq. (13), $\gamma_{I}$ is the nuclear gyromagnetic ratio, $\mu_{0}$ is the permeability of space, and $r_{I S}$ is the length of the $I-S$ interspin vector. The functions $F_{q}^{(1)}(\theta, \varphi)$ are first rank spherical tensors constructed from the electron spin variables, $S_{p}^{(1)}$ and the spherical harmonics, $Y_{2, p}(\theta, \varphi)$, which describe the orientation of the interspin $I-S$ vector in the laboratory coordinate frame:

$$
\begin{aligned}
F_{m}^{(1)}(\theta, \varphi)= & (12 \pi / 5)^{1 / 2} \sum_{p, q=-1}^{+1}(-1)^{1-q} \\
& \times\left[\begin{array}{ccc}
1 & 2 & 1 \\
p & q & -m
\end{array}\right] S_{p}^{(1)} Y_{2, q}(\theta, \varphi) .
\end{aligned}
$$

The symbols in square brackets are $3-j$ symbols. The physical significance of the operators $F_{m}^{(1)}$ is evident from the form of Eq. (12), which expresses $H_{\text {dip }}$ in terms of the scalar product, $\vec{F} \cdot \vec{I}$ [which is written in spherical tensor form in Eq. (12)]. In the semiclassical picture, the dipolar energy is $E_{\text {dip }}=-\vec{B}_{\text {dip }} \cdot \vec{\mu}_{I}$. The $F_{q}^{(1)}(\theta, \varphi)$ are thus proportional to the spherical components of the operator that represents the local dipolar field of $S$ at the nuclear spin $I$.

From Eqs. (12)-(14), the expression for the dipolar $R_{1}$ NMR-PRE is

$$
\begin{aligned}
\left(T_{1 p}\right)^{-1}= & \left(-3^{-1} \kappa^{2} r_{I S}^{-6}\right) \\
& \times \int_{0}^{\infty}\left\{\overline{\left\langle F_{-1}^{(1)}(t) F_{+1}^{(1)}(0)\right\rangle} e^{i \omega_{i} t}+\text { c.c. }\right\} d t .
\end{aligned}
$$

The dipolar TCF, $G_{\text {dip }}(t)$, is defined as

$$
G_{\mathrm{dip}}(t)=\overline{\left\langle F_{p}^{(1)}(t) F_{-p}^{(1)}(0)\right\rangle} \quad(p=0, \pm 1) .
$$

This quantity describes the motion (both coherent and stochastic) of the dipolar field of the electron spin $S$ at the nuclear spin $I$. [The notation of Eqs. (15) and (16) omits the $\{\theta, \varphi\}$ dependence of the $F_{q}^{(1)}(\theta, \varphi ; t)$, since this dependence is eliminated from $G_{\text {dip }}(t)$ when the spatial average is calculated.] 


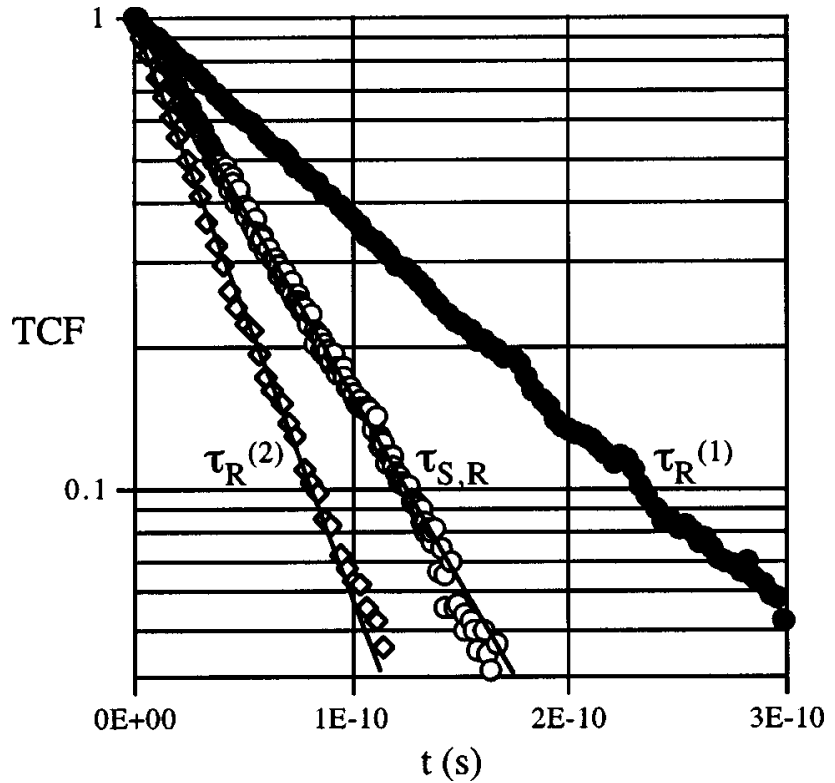

FIG. 1. Decay of the normalized spin TCF, $G_{Z}(t)$, in the cylindrical zfs limit for $S=1$. Also shown are the simulated decays of molecule-fixed firstand second-rank tensors $\left(\tau_{R}^{(1)}\right.$ and $\left.\tau_{R}^{(2)}\right)$. The calculations assumed zfs parameters, $D=1 \mathrm{~cm}^{-1}$ and $E=0 \mathrm{~cm}^{-1}$, with $B_{0}=0$ and $\tau_{R}^{(1)}=100 \mathrm{ps}$. Decays are averaged over 1472 trajectories.

\section{RESULTS}

\section{A. ZFS-limit behavior}

Figure 1 shows a plot of the spin TCF, $G_{Z}(t)$, for a spin $S=1$ in the cylindrical zfs limit $\left(D=1 \mathrm{~cm}^{-1}, E=0, B_{0}\right.$ $=0 \mathrm{~T}$ ). Also shown on the plot are the simulated decays of a molecule-fixed vector, for which the decay constant is $\tau_{R}^{(1)}$, and a molecule-fixed second-rank tensor $\left(\tau_{R}^{(2)}\right)$. The $\sigma_{\phi}$ parameter of the simulation was calculated using Eq. (8) with $\tau_{R}^{(1)}=100 \mathrm{ps}$ and $n_{J}=30$. All three decays are exponential, and the ratio of decay constants, $\tau_{R}^{(2)} / \tau_{R}^{(1)}=3^{-1}$, obeys the well known relationship, $\tau_{R}^{(\ell)} \propto[\ell(\ell+1)]^{-1}$, for $\ell$ th-rank molecule-fixed tensors. The simulated decay constant of $G_{Z}(t)$ is thus intermediate between $\tau_{R}^{(1)}$ and $\tau_{R}^{(2)}$, approximately,

$$
\tau_{S, Z}=0.53 \tau_{R}^{(1)} .
$$

This result reflects the following physical situation. In the zfs limit, when the zfs tensor is cylindrical $(E=0)$, $G_{Z}(t)$ is a constant of the motion in the absence of molecular reorientation. (This fact is evident from Eq. (4), since $\left[H_{\mathrm{zfs}}^{\circ}, \hat{S}_{z}\right]=0$ when $E=0$.) If the spin were a classical vector, $G_{Z}(t)$ would decay according to $\tau_{R}^{(1)}$. However, the quantum mechanical spin system undergoes transitions as the zfs tensor reorients, leading to a decay that is faster than $\tau_{R}^{(1)}$. As an illustration, we consider the transition from the first to the second interval in a random walk trajectory. For $t_{0}<t<t_{1}$, $\left\langle\hat{S}_{z}\right\rangle$ and $G_{Z}(t)$ are constants of the motion. At the jump, the zfs tensor reorients, and the spin motion is requantized along the new principal axis of the zfs tensor. For $t_{1}<t<t_{2}$, $\left(\left\langle\hat{S}_{z}\right\rangle\right)^{\prime}$ and $\left[G_{Z}(t)\right]^{\prime}$, evaluated in the new eigensystem of $\left(H_{\mathrm{zfs}}^{\circ}\right)^{\prime}$, are also constants of the motion. However, the change in the axes of spin quantization leads to transitions

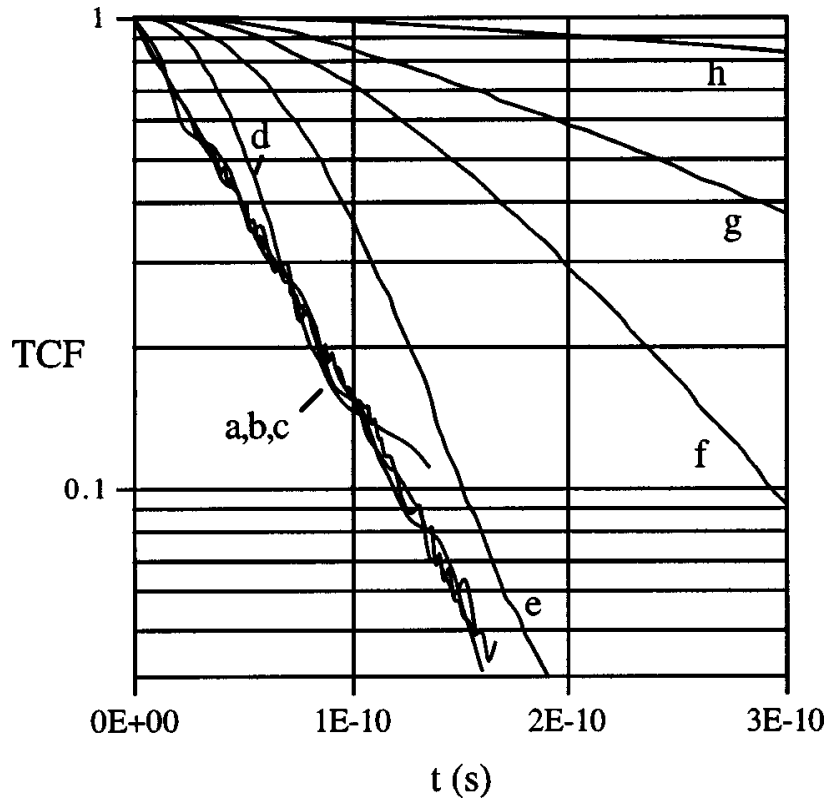

FIG. 2. Dependence of the electron spin time correlation function $G_{Z}(t)$ on the cylindrical zfs parameter $D$. Curves (a)-(h) were calculated assuming $D=10,3,1,0.3,0.17,0.1,0.07$, and $0.03 \mathrm{~cm}^{-1}, \tau_{R}^{(2)}=33 \mathrm{ps}$. This range of $D$ corresponds to $63 \geqslant \omega_{D} \tau_{R}^{(2)} \geqslant 0.19$. Other parameters are the same as for Fig. 1.

out of the original eigenstates of $H_{\mathrm{zfs}}^{\circ}$ and these transitions represent a loss of spin memory in addition to that due to simple reorientation of the $z$ axis. In the Sudden Approximation, the probability of transition out of the eigenstate, $\left|i_{n}\right\rangle$, of $\left(H_{\mathrm{zfs}}^{\circ}\right)^{(n)}$ during the $n$th interval, into eigenstate $\left|j_{n+1}\right\rangle$, of the Hamiltonian, $\left(H_{\mathrm{zfs}}^{\circ}\right)^{(n+1)}$, during interval $n+1$, is given by $^{32}$

$$
P\left(i_{n} \rightarrow j_{n+1}\right)=\left|\left\langle i_{n} \mid j_{n+1}\right\rangle\right|^{2} .
$$

It is an interesting result of the simulation of Fig. (1) that the zfs limit decay due to spin transitions is approximately equal to the decay due to vector reorientation, i.e.,

$$
\tau_{S, R} \approx \tau_{R}^{(1)} / 2 \text {. }
$$

A similar result was found by Bertini, Luchinat, and Kowalewski ${ }^{34}$ in calculations of the NMR-PRE produced by a uniaxial $S=1$ spin system reorienting rapidly in the zfs limit.

Figure 2 shows the effect of motional averaging of the zfs energy levels on the decay of $G_{Z}(t)$. The level structure due to $H_{\mathrm{zfs}}^{\circ}$ is well defined only when $\omega_{D} \tau_{R}^{(2)}>1$. More rapid reorientation averages and collapses the level structure. In the limit where $\omega_{D} \tau_{R}^{(2)} \ll 1$, the decay of $G_{Z}(t)$ is expected to become long, approaching infinity in the limit of short $\tau_{R}^{(2)}$. These phenomena are illustrated by the simulations in Fig. 2, which shows the effect of varying $D$ with constant $\tau_{R}^{(2)}$ $=33 \mathrm{ps}$. The onset of reorientational collapse of the level structure occurs in the vicinity of $\omega_{D} \tau_{R}^{(2)}=1$, which in the figure corresponds to a zfs parameter, $D=0.17 \mathrm{~cm}^{-1}$ (curve $e$ ). For $D<0.3 \mathrm{~cm}^{-1}$ (curves $f, g, h$ ), the decay slows and becomes non-linear, as expected. When $\omega_{D} \tau_{R}^{(2)}>2$, the decay is almost independent of $D(a, b, c)$ and the decay constant is given by Eq. (17). 
a)

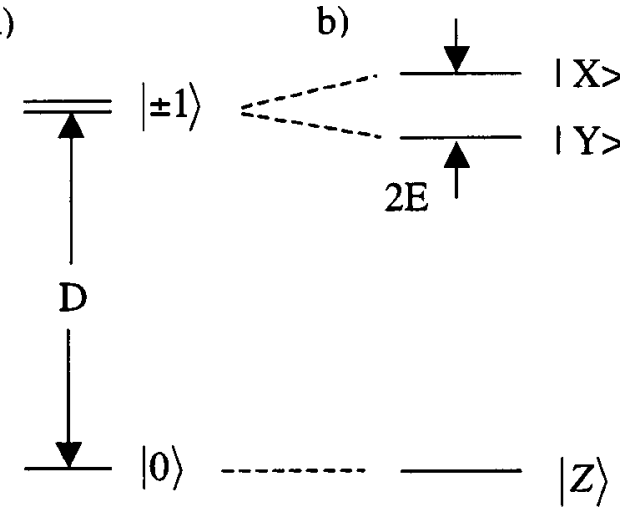

FIG. 3. Energy levels and spin eigenfunctions for $S=1$ in the zfs limit. The diagrams assume a cylindrical (a) and orthorhombic (b) zfs tensor, with positive $D$ and $E$.

These results describe $S=1$ when the zfs tensor is cylindrical. The presence of an orthorhombic term, $E 0$, in the zfs tensor forces a cartesian polarization on the spin wave functions and splits the $m_{S}= \pm 1$ non-Kramers doublet. The change in the level diagram and in the labeling of spin wave functions is shown in Fig. 3. When $E \neq 0,\left[H_{\mathrm{zfs}}^{\circ}, \hat{S}_{z}\right] \neq 0$, and $G_{Z}(t)$ is no longer a constant of the motion; rather it is driven into coherent oscillation at the non-Kramers doublet splitting, $2 \omega_{E}=4 \pi c E$. The oscillation in $G_{Z}(t)$ increases the rate of loss of phase memory relative to the cylindrical zfs case. Figure 4 shows simulated spin decays, calculated for physical conditions equivalent to those of Fig. 1 except with a non-zero $E$ value. The orthorhombicity-induced oscillation of $\left\langle\hat{S}_{z}\right\rangle$ introduces nonlinearity into the decay and accelerates it (Fig. 4). The maximum physical value of $E$ is $E=D / 3$ (larger values of $E$ are usually described by redefin-

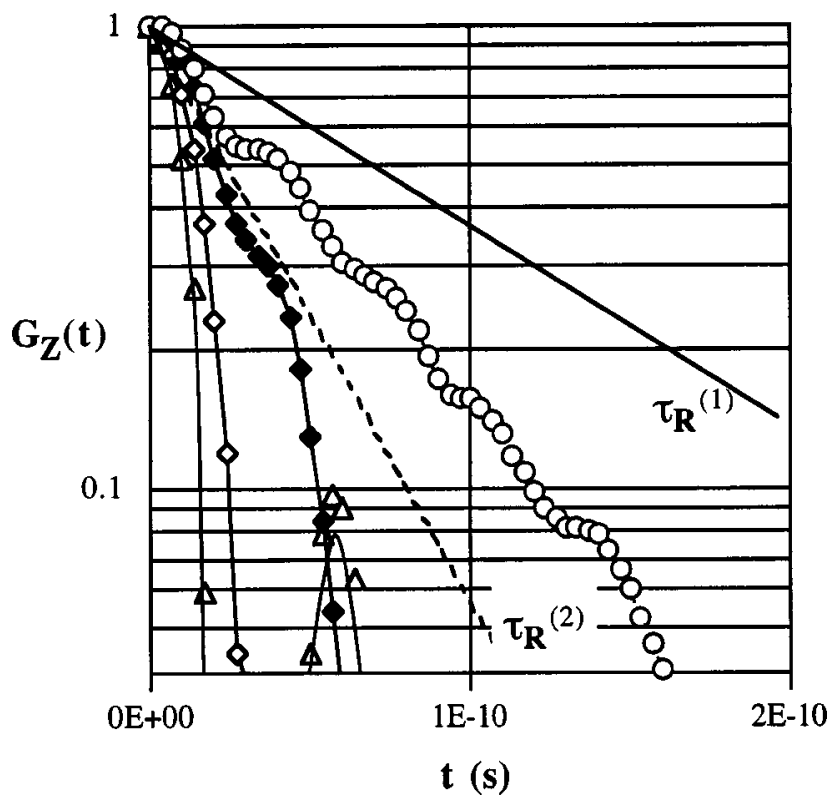

FIG. 4. Simulations of the spin TCF for $S=1, D=1 \mathrm{~cm}^{-1}$, with four values of $E / D: E / D=0$ (circles)); $E / D=0.1$ (filled diamonds); $E / D=0.2$ (unfilled diamonds); $E / D=0.3$, (unfilled triangles). Decays of $\tau_{R}^{(1)}$ and $\tau_{R}^{(2)}$ are shown as solid and dashed lines, respectively.

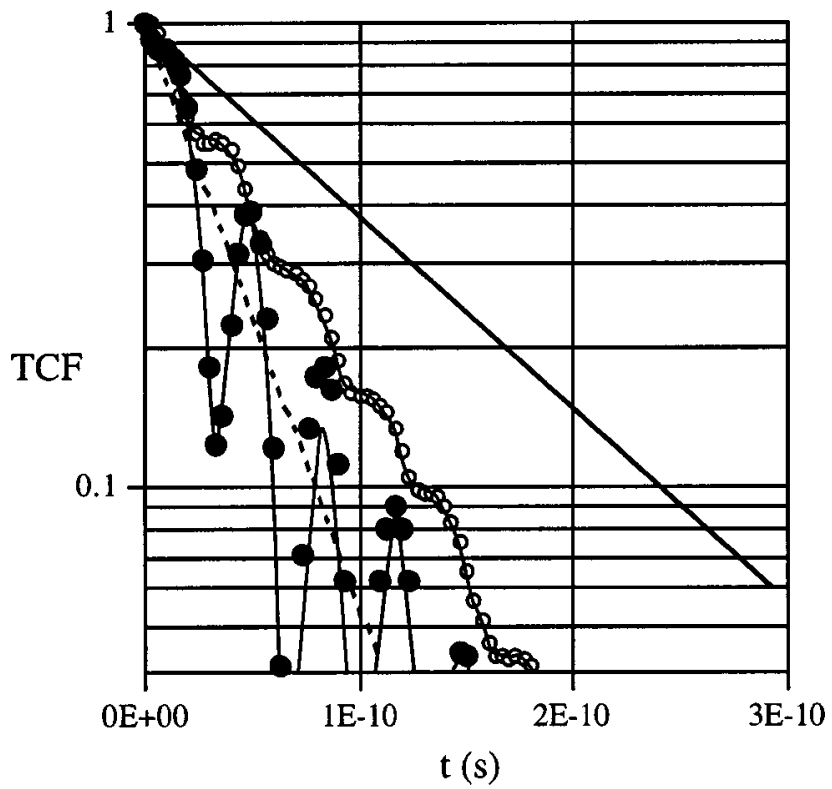

FIG. 5. Decay of $G_{Z}(t)$ (open circles) and $G_{\text {dip }}(t)$ (filled circles) in the vicinity of the zfs limit: $\omega_{Z} / \omega_{D}=0.008$. The calculations assumed that $D$ $=1 \mathrm{~cm}^{-1}$ and $B_{0}=0.01 \mathrm{~T}$, with other parameters the same as Fig. 3. Decays are also shown for $\tau_{R}^{(1)}$ (solid line) and $\tau_{R}^{(2)}$ (dashed line).

ing the coordinate system), at which point the 1/e decay time of $G_{Z}(t)$ is shorter by a factor of roughly 5 relative to the $E=0$ situation.

\section{B. Comparison of the motions of $G_{z}(t)$ and $G_{\text {dip }}(t)$ in the intermediate regime}

Equations (2) and (16) define the two TCFs, $G_{Z}(t)$ and $G_{\text {dip }}(t)$, the first of which describes the electron spin motion, the second, the motion of the local dipolar field of electron spin $S$ at the nuclear spin $I$. It is $G_{\text {dip }}(t)$ that is directly related to the NMR-PRE through Eq. (15). We expect, on physical grounds, that $G_{Z}(t)$ and $G_{\mathrm{dip}}(t)$ will coincide in the zfs-limit, since in that situation the location of the $I$ spin is fixed with respect to the quantization axes of the electron spin motion. Reorientation of the zfs principal axis system (zfs-PAS) does not alter dipolar field of $S$ at $I$. In the Zeeman limit, the situation is very different: The electron spin motion is quantized along the laboratory magnetic field and has no fixed relation to the interspin vector. In this limit, the reorientational correlation time is that of a second-rank moleculefixed tensor $\tau_{R}^{(2)}$.

Figures 5-7 show the decay of $G_{Z}(t)$ and $G_{\text {dip }}(t)$ as the spin system passes between the zfs and Zeeman limits. The ratio $\omega_{Z} / \omega_{D}$, where $\omega_{Z}$ is the electron Larmor frequency, is useful for tracking the passage of the spin system through the intermediate regime, which is centered, approximately, where $\omega_{Z} / \omega_{D} \approx 1$. Figure 5 shows simulations performed in the vicinity of the zfs limit. $G_{\mathrm{dip}}(t)$ exhibits substantial coherent oscillation, but the stochastic decay of the envelope coincides with the that of $G_{Z}(t)$. Thus, in the zfs limit, both $G_{Z}(t)$ and $G_{\mathrm{dip}}(t)$ decay with the time constant given by Eq. (17). Figure 6 shows the TCF decay in the intermediate regime of field strengths $\left(\omega_{Z} / \omega_{D}=0.24\right)$. The decay of the 


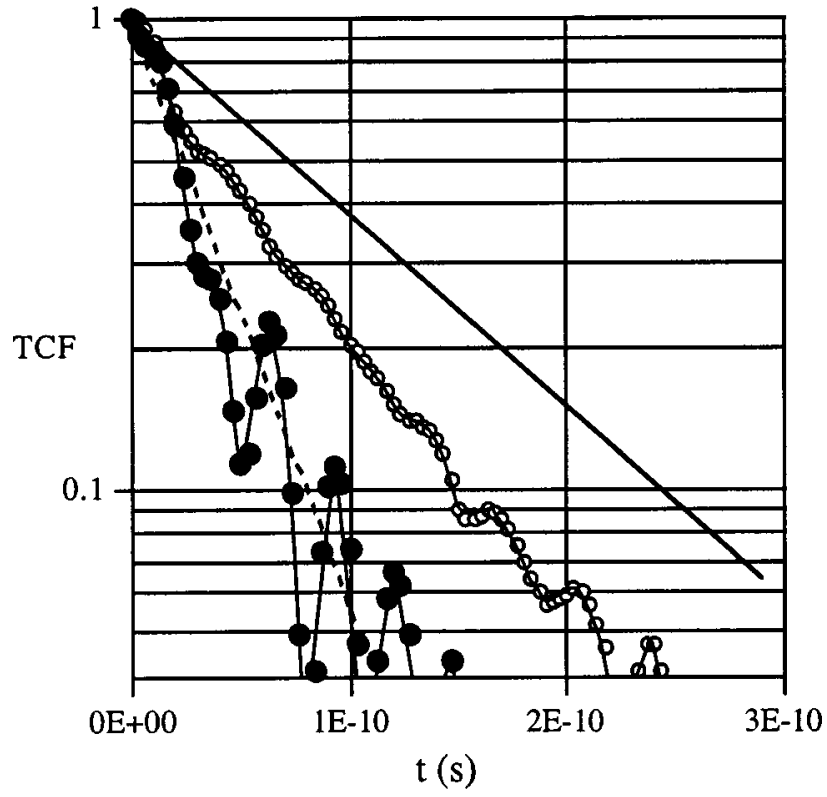

FIG. 6. Decay of $G_{Z}(t)$ (open circles) and $G_{\text {dip }}(t)$ (filled circles) in the intermediate regime of field strengths: $\omega_{Z} / \omega_{D}=0.24$. The calculations assumed that $D=1 \mathrm{~cm}^{-1}, B_{0}=0.3 \mathrm{~T}$, with other parameters the same as Fig. 3. Decays are also shown for $\tau_{R}^{(1)}$ (solid line) and $\tau_{R}^{(2)}=$ (dashed line).

spin TCF, $G_{Z}(t)$, slows, while that of the dipolar TCF, $G_{\text {dip }}(t)$, becomes faster relative to the zfs limit.

Figure 7 shows the progress toward the Zeeman limit. The decay constant $\tau_{S, Z}$ of the spin TCF becomes very long compared to the zfs-limit value of Eq. (17) while the decay constant of $G_{\mathrm{dip}}(t)$ shortens, approaching $\tau_{R}^{(2)}$. The progress between the zfs and Zeeman limits is plotted in Fig. 8, which shows the (1/e) decay time as a function of $\omega_{Z} / \omega_{D}$ (both

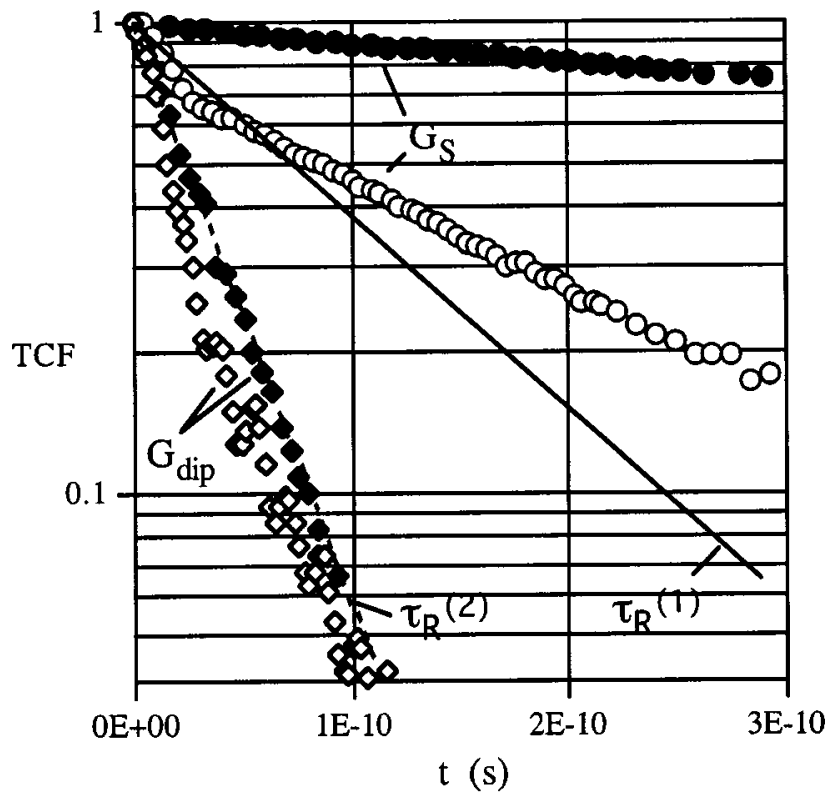

FIG. 7. Decay of $G_{Z}(t)$ (circles) and $G_{\mathrm{dip}}(t)$ (triangles) in the vicinity of the Zeeman limit and in the intermediate regime: $\omega_{Z} / \omega_{D}=2.4$ (solid symbols) and 0.81 (open symbols). The calculations assumed that $D=1 \mathrm{~cm}^{-1}$ with $B_{0}=3 \mathrm{~T}$ (solid symbols) or $1 \mathrm{~T}$ (open symbols), with other parameters the same as in Fig. 1. Decays are also shown for $\tau_{R}^{(1)}$ (solid line) and $\tau_{R}^{(2)}=($ dashed line $)$

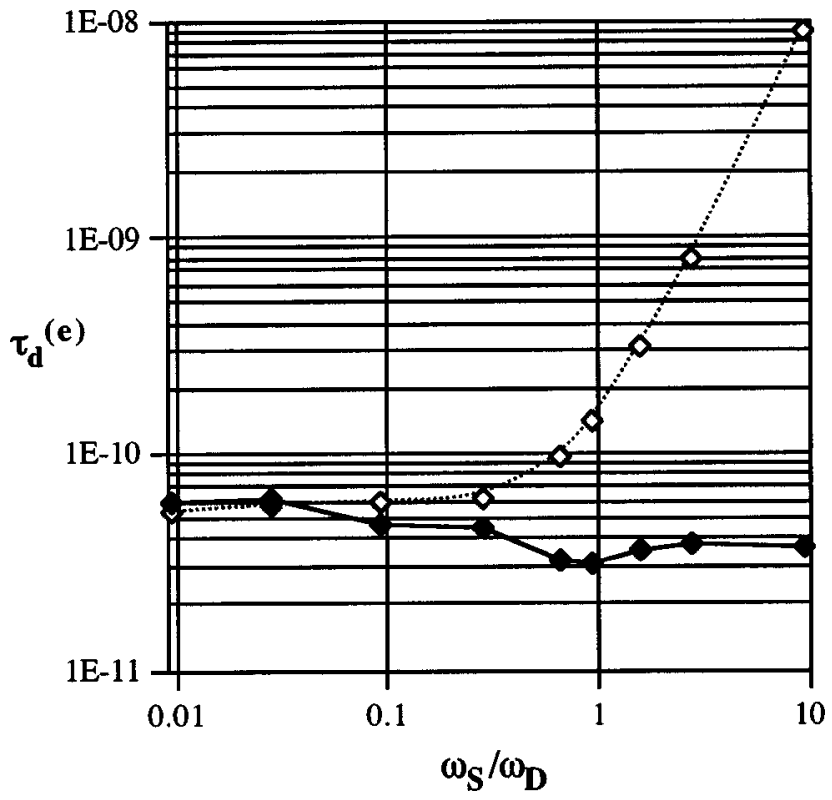

FIG. 8. Estimated 1/e decay times of the spin TCF $\left[G_{Z}(t)\right.$, unfilled symbols], and the dipolar TCF, [ $G_{\text {dip }}(t)$, filled], in passing between the Zeeman and zfs limits.

decays are nonexponential in the intermediate regime). In these simulations, $B_{0}$ varied from 0.01 to $10 \mathrm{~T}$ with $D$ $=1 \mathrm{~cm}^{-1}, E=0$.

The Zeeman-limit behavior is also shown in Fig. 9, which contains a plot of the spin decay rate, $\left(\tau_{S, Z}\right)^{-1}$, as a function of $D$ at constant $B_{0}=1 \mathrm{~T}$. The variation of $D$ corresponds to the range $0.21 \geqslant\left(\omega_{D} / \omega_{Z}\right)$, i.e., the approach to the Zeeman limit. In this region, the decay rate is proportional to $\omega_{D}^{2}\left(\tau_{R}^{(2)}\right)$.

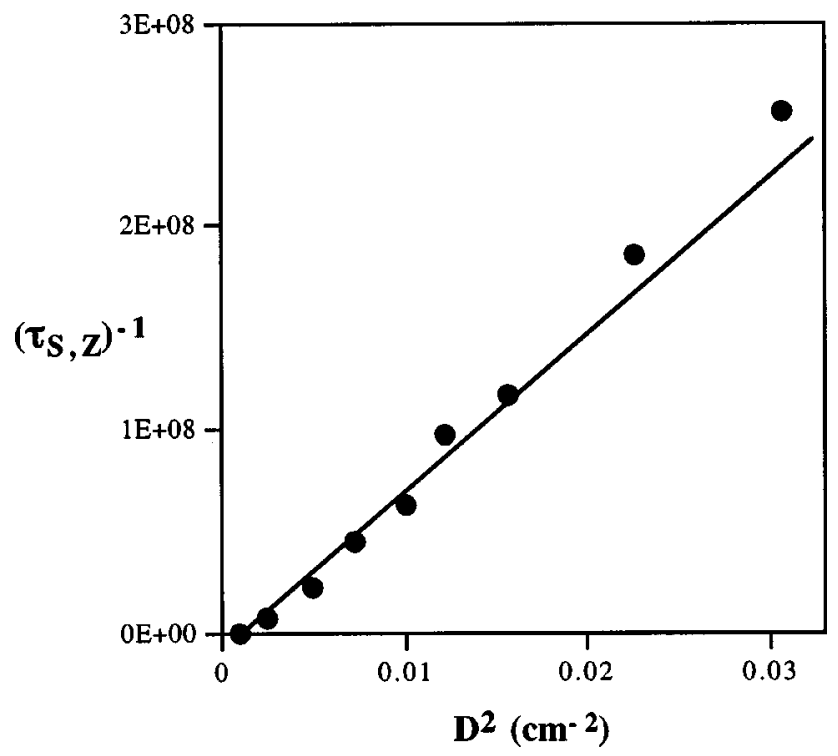

FIG. 9. Decay of the spin TCF, $G_{Z}(t)$, in the vicinity of the Zeeman Limit. SD simulations were performed at constant Zeeman field strength, $B 0$ $=1 \mathrm{~T}$, with variation of $D$. Other parameters are given in the legend of Fig. 1. 


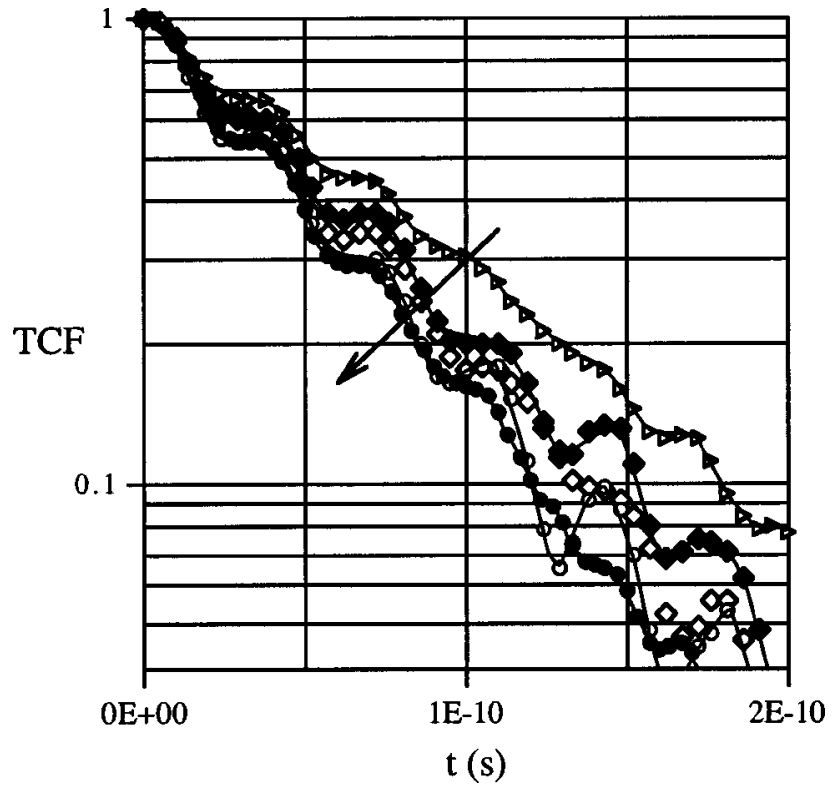

FIG. 10. Effect of varying $n_{J}$ (number of reorientational jumps per $\tau_{R}^{(1)}$ interval) on the electron spin TCF. TCFs were calculated assuming $n_{J}=4,6$, $10,15,30$, increasing with the arrow. Other parameters are the same as in Fig. 1.

\section{Dependence of the spin motion on reorientational jump frequency}

The simulations described above were calculated using a random walk model with $n_{J}=30$ reorientational jumps per $\tau_{R}^{(1)}$ period. To examine whether the results depend on the reorientational jump frequency, simulations were performed over a range of values, $4 \leqslant n_{J} \leqslant 30$, with results that are shown in Fig. 10. The spin motion was nearly independent of $n_{J}$ for $n_{J} \geqslant 15$.

\section{SUMMARY OF RESULTS}

The objective of this study is to characterize the electron spin mechanism due to zfs tensor reorientation in the intermediate and zfs-limit regimes for $S=1$. Based on the results of SD simulations, the behavior of the spin and dipolar TCFs, $G_{Z}(t)$ and $G_{\mathrm{dip}}(t)$, can be summarized as follows:

(A) When the zfs tensor is cylindrical $(E=0)$ :

(1) The zfs-limit decays of the spin and dipolar TCFs occurs with the same time constant, $\tau_{d e c} \approx 0.53 \tau_{R}^{(1)}$ [Eq. (17)]. This decay constant is independent of the magnitude of $D$ and of the jump frequency, $n_{J}$.

(2) Molecular reorientation that is rapid on the timescale of the inverse level splittings $\left(\omega_{D} \tau_{R}^{(2)} \leqslant 1\right)$ collapses the zfs level structure, leading to a lengthening of the spin decay time, $\tau_{S, Z} \rightarrow \infty$ in the fast reorientation limit.

(3) In the vicinity of the Zeeman limit $\left(\omega_{Z} / \omega_{D} \gg 1\right)$, the decay constant of $G_{Z}(t)$ becomes long, the inverse decay constant tending to zero as $D^{2}$; thus, in passing from the zfs limit to the Zeeman limit $\tau_{S, Z}$ varies between limiting values, $0.53 \tau_{R}^{(1)} \leqslant \tau_{S, Z} \leqslant \infty$.

(4) The dipolar TCF, $G_{\text {dip }}(t)$, is related more directly to the NMR-PRE than the spin TCF, $G_{Z}(t)$. In the zfs limit, the decay constants of $G_{Z}(t)$ and $G_{\mathrm{dip}}(t)$ are equal. In the Zee- man limit, the decay constant of $G_{\mathrm{dip}}(t)$ approaches $\tau_{R}^{(2)}$ as required by Zeeman-limit. Theory, while the decay rate of $G_{Z}(t)$ approaches zero.

(B) The presence of an orthorhombic term $(E \neq 0)$ in the zfs tensor strongly influences both $G_{Z}(t)$ and $G_{\text {dip }}(t)$. The zfs limit decay shortened several fold in simulations when $E / D$ increased from zero to its maximum value of $1 / 3$.

\section{DISCUSSION}

\section{A. Dependence of the results on the diffusional model}

The zfs limit decay kinetics described above are not very sensitive to the reorientational jump frequency (Fig. 10), but they do depend on the assumption that reorientational motion occurs as rapid jumps, rather than as continuous motions. In the limiting situation of slow, continuous reorientation, the motion of the spin system is described by the adiabatic approximation rather than by the sudden approximation. The motion of $\left\langle S_{Z}\right\rangle$ is then locked to the zfs principal axes, and reorientation does not induce spin transitions. In this case, decay of $G_{Z}(t)$ is described by $\tau_{R}^{(1)}$, as for a classical vector. However, it seems likely that in most practical cases the elementary rotational motions of Brownian diffusion will be rapid compared to the inverse spin transition frequencies, in which case the random walk model and the zfs limit decay time of Eq. (17) provide a better description.

It is interesting that the calculations by Bertini, Luchinat, and Kowalewski ${ }^{34}$ of the NMR-PRE produced by a reorienting $S=1$ spin system in the zfs limit likewise gave the result of Eq. (17), although that study described reorientation by the classical diffusion equation rather than by the random walk model used here. It is expected that $\tau_{S, Z}$ will be less than $\tau_{R}^{(1)}$ when the elementary diffusive motions are rapid compared to the motions of the spin system, but the quantitative result of Eq. (17) has not yet been shown to be model independent. However, the agreement of these two studies suggests that this result may be a general one when diffusive motions are rapid.

\section{B. Relation to NMR-PRE}

We consider the question of how these findings bear on calculations of the NMR-PRE. The Zeeman-limit situation is straightforward since $H_{S}$ is time independent and SBM Theory applies. When a permanent zfs interaction is present, however, $H_{S}(t)$ and the spin wavefunctions are timedependent, which introduces considerable complexity into the theory. The SD simulation methods described above $\mathrm{e}^{27,30,33,35}$ and the stochastic Liouville formalism developed in Sweden ${ }^{36-39}$ can be used in the general situation.

An intermediate level of theory may be appropriate when $H_{\mathrm{zfs}}^{\circ}$ is significant in magnitude but is effectively time independent due to slow Brownian reorientation. This level of theory, often called "Slow Reorientation", has been developed both in this laboratory ${ }^{40-42}$ and by workers in Florence ${ }^{43}$ and Sweden. ${ }^{37,44}$ The slow reorientation approach can improved to account for a portion of the reorientational time dependence by inserting a factor, $\exp \left(-t / \tau_{R}^{(2)}\right)$, into 
$G_{\text {dip }}(t)$ while ignoring the time dependence of $H_{S}(t)$ in Eq. (2) ${ }^{30,45,46}$ This procedure is intended to describe the motion of the interspin vector $\vec{r}_{I S}$ while neglecting the motion of $H_{S}(t)$. The approach is attractive in that it permits relatively rapid and simple calculations of the NMR-PRE while accounting in part for both the zfs interaction and reorientational motion. Prior work ${ }^{30,44}$ has indicated that these contributions are substantial.

However, the question arises as to what reorientational correlation time is appropriate in slow reorientation theory. In the Zeeman limit, it is clearly $\tau_{R}^{(2)}$, but this quantity is only appropriate when both spins $I$ and $S$ have Zeeman quantization. When the $S$ spin does not have a Zeeman quantization, the reorientational time dependence of the $I-S$ dipolar interaction is described by $G_{\mathrm{dip}}(t)$. This quantity describes the coupled motions of the electron spin (including motions driven both by reorientation of the zfs-PAS and by spin transitions) and the motion of $\vec{r}_{I S}$. The correct reorientational correlation time for slow reorientation theory is the magnetic field-dependent quantity, $\tau_{S \text {,dip }}$, which, for $S=1$ and a cylindrical zfs tensor, is plotted in Fig. 8.

For the conditions described by the figure, $\tau_{S \text {, dip }}$ varies between limits of $\tau_{R}^{(2)}$ in the Zeeman limit and that given by Eq. (17) in the zfs limit. Thus the use of $\tau_{R}^{(2)}$ in slow reorientation theory, while physically inappropriate outside of the Zeeman limit, introduces only modest quantitative error $(\leqslant 30 \%)$, which could be considered a reasonable approximation. However, the presence of rhombicity in the zfs tensor can have a large (as much as several fold) influence on the decay of $G_{\text {dip }}(t)$, as shown in Fig. 4. The magnitude of zfs rhombicity is usually unknown (except when excluded by symmetry), and thus in most cases, our estimate of $\tau_{S \text {, dip }}$ is rather crude. For $S=1, \tau_{S \text {, dip }}$ is no larger than the value given by Eq. (17) but may be several fold shorter.

The dramatic effect of zfs rhombicity on the spin motion of integer spins results from rhombicity-induced splitting of $m_{S}= \pm 1$ non-Kramers doublet. ${ }^{39,47-49}$ For half-integer spins, the Kramers doublets are unsplit by zfs couplings of all orders, and the motion of $G_{\text {dip }}(t)$ is less affected by low symmetry terms in the zfs tensor. Thus, it seems likely that the inherent uncertainty in $\tau_{S \text {,dip }}$ is much smaller for half-integer spins than for integer spins, although further study of this point is needed.

\section{ACKNOWLEDGMENT}

This material is based upon work supported by the National Science Foundation under Grant No. CHE-0209616.

${ }^{1}$ J. H. Van Vleck, Phys. Rev. 57, 426 (1940).

${ }^{2}$ N. Bloembergen and L. O. Morgan, J. Chem. Phys. 34, 842 (1961).

${ }^{3}$ A. Carrington and G. R. Luckhurst, Mol. Phys. 8, 125 (1964).

${ }^{4}$ M. Rubinstein, A. Baram, and Z. Luz, Mol. Phys. 20, 67 (1971).

${ }^{5}$ B. B. Garrett and L. O. Morgan, J. Chem. Phys. 44, 890 (1966).

${ }^{6}$ G. R. Luckhurst and G. F. Pedulli, Mol. Phys. 22, 931 (1971).
${ }^{7}$ P.-O. Westlund, N. Benetis, and H. Wennerstrom, Mol. Phys. 61, 177 (1987).

${ }^{8}$ J. Svoboda, T. Nilsson, J. Kowalewski, P.-O. Westlund, and P. T. Larsson, J. Magn. Reson., Ser. A 121, 108 (1996).

${ }^{9}$ P.-O. Westlund and P. T. Larsson, Acta Chem. Scand. 45, 11 (1991).

${ }^{10}$ P.-O. Westlund, J. Chem. Phys. 108, 4945 (1998).

${ }^{11}$ I. Bertini, J. Kowalewski, C. Luchinat, T. Nilsson, and G. Parigi, J. Chem. Phys. 111, 5795 (1999).

${ }^{12}$ R. Sharp and L. Lohr, J. Chem. Phys. 115, 5005 (2001).

${ }^{13}$ R. Sharp, J. Magn. Reson. 154, 269 (2002).

${ }^{14}$ A. Hudson and G. R. Luckhurst, Mol. Phys. 16, 395 (1969).

${ }^{15}$ A. Hudson and J. W. E. Lewis, Trans. Faraday Soc. 66, 1297 (1970).

${ }^{16}$ A. D. McLachlan, Proc. R. Soc. London 280, 271 (1964).

${ }^{17}$ H. Levanon, S. Charbinsky, and Z. Luz, J. Chem. Phys. 53, 3056 (1970).

${ }^{18}$ S. Rast, A. Borel, L. Helm, E. Belorizky, P. H. Fries, and A. E. Merbach, J. Am. Chem. Soc. 123, 2637 (2001).

${ }^{19}$ S. Rast, P. H. Fries, and E. Belorizky, J. Chem. Phys. 113, 8724 (2000).

${ }^{20}$ R. Sharp, Nucl. Magn. Reson., Spec. Period. Rep. 30, 477 (2001); 32, 473 (2003).

${ }^{21}$ E. Toth, L. Helm, and A. E. Merbach, Comprehensive Coord. Chem. II 9 , 841 (2004).

${ }^{22}$ N. Benetis, J. Kowalewski, L. Nordenskiold, H. Wennerstrom, and P.-O. Westlund, Mol. Phys. 48, 329 (1983).

${ }^{23}$ N. Benetis, J. Kowalewski, L. Nordenskiold, H. Wennerstrom, and P.-O. Westlund, J. Magn. Reson. (1969-1992) 58, 261 (1984).

${ }^{24}$ P.-O. Westlund, H. Wennerstrom, L. Nordenskiold, J. Kowalewski, and N. Benetis, J. Magn. Reson. (1969-1992) 59, 91 (1984).

${ }^{25}$ N. Benetis, J. Kowalewski, L. Nordenskiold, H. Wennerstrom, and P.-O. Westlund, Mol. Phys. 50, 515 (1983).

${ }^{26}$ J. Kowalewski, L. Nordenskiold, N. Benetis, and P.-O. Westlund, Prog. Nucl. Magn. Reson. Spectrosc. 17, 141 (1985).

${ }^{27}$ An earlier version of the program called SpinDyn is described by S. M. Abernathy and R. R. Sharp, J. Chem. Phys. 106, 9032 (1997).

${ }^{28}$ E. N. Ivanov, Zh. Eksp. Teor. Fiz. 45, 1509 (1963) [Sov. Phys. JETP 18, 1041 (1964).

${ }^{29}$ M. Odelius, C. Ribbing, and J. Kowalewski, J. Chem. Phys. 103, 1800 (1995).

${ }^{30}$ J. Miller, N. Schaefle, and R. Sharp, Magn. Reson. Chem. 41, 806 (2003).

${ }^{31}$ A. Messiah, Quantum Mechanics (Wiley, New York, 1962), Chap. XVII.

${ }^{32}$ S. M. Blinder, Foundations of Quantum Mechanics (Academic, New York, 1974), Chap. 7.

${ }^{33}$ R. Sharp, L. Lohr, and J. Miller, Prog. Nucl. Magn. Reson. Spectrosc. 38, 115 (2001).

${ }^{34}$ I. Bertini, C. Luchinat, and J. Kowalewski, J. Magn. Reson. (1969-1992) 62, 235 (1985).

${ }^{35}$ J. C. Miller and R. R. Sharp, J. Phys. Chem. A 104, 4889 (2000).

${ }^{36}$ P.-O. Westlund, in Dynamics of Solutions and Fluid Mixtures by NMR, edited by J. J. Delpuech (Wiley, New York, 1995), p. 173.

${ }^{37}$ T. Nilsson and J. Kowalewski, J. Magn. Reson. 146, 345 (2000).

${ }^{38}$ D. Kruk, T. Nilsson, and J. Kowalewski, Phys. Chem. Chem. Phys. 3, 4907 (2001)

${ }^{39}$ R. Nilsson, J. Svoboda, P.-O. Westlund, and J. Kowalewski, J. Chem. Phys. 109, 6364 (1998).

${ }^{40}$ R. R. Sharp, J. Chem. Phys. 93, 6921 (1990).

${ }^{41}$ R. R. Sharp, J. Magn. Reson. (1969-1992) 100, 491 (1992).

${ }^{42}$ R. Sharp, S. M. Abernathy, and L. L. Lohr, J. Chem. Phys. 107, 7620 (1997).

${ }^{43}$ I. Bertini, O. Galas, C. Luchinat, and G. Parigi, J. Magn. Reson., Ser. A 113, 151 (1995).

${ }^{44}$ J. Kowalewski, C. Luchinat, T. Nilsson, and G. Parigi, J. Phys. Chem. A 106, 7376 (2002).

${ }^{45}$ D. Kruk and J. Kowalewski, JBIC, J. Biol. Inorg. Chem. 8, 512 (2003).

${ }^{46}$ T. Nilsson and J. Kowalewski, Mol. Phys. 98, 1617 (2000).

${ }^{47}$ R. R. Sharp, J. Chem. Phys. 98, 6092 (1993).

${ }^{48}$ S. M. Abernathy, J. C. Miller, L. L. Lohr, and R. R. Sharp, J. Chem. Phys. 109, 4035 (1998).

${ }^{49}$ J. C. Miller, L. L. Lohr, and R. R. Sharp, J. Magn. Reson. 148, 267 (2001). 